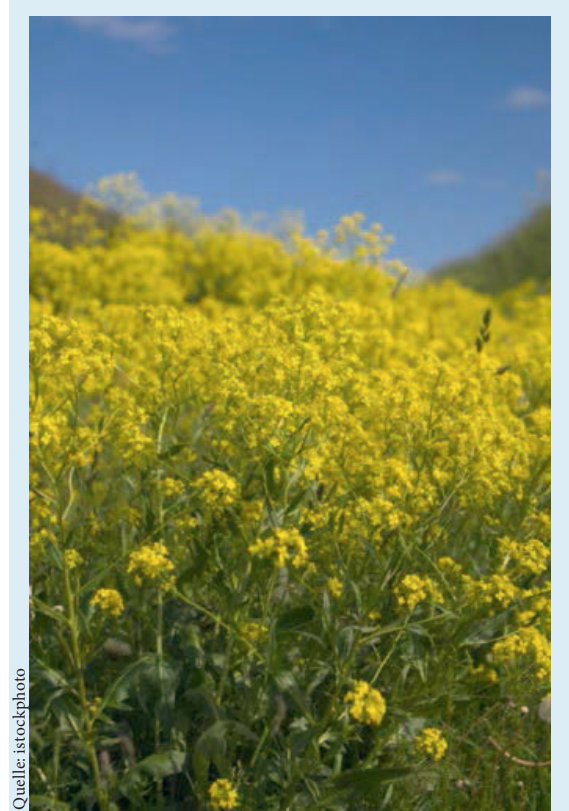

\section{Forschung und Entwicklung in der Phytotherapie}

Immer besser, immer wirksamer und immer alles neu: Das ist das Credo in der Medizin - doch in der Phytotherapie hapert es diesbezüglich. Innovationen auf dem Markt gibt es selten, und schuld daran sind die Vorschriften der Zulassungsbehörden. Dass das nicht immer so war, berichtet der Leiter des Pharmaziehistorischen Museums in Basel, Dr. Michael Kessler, im Expertengespräch. Und dass an den Universitäten sehr wohl innovativ an neuen Phytotherapeutika geforscht wird, erfahren Sie im Expertengespräch mit Prof. Dr. Matthias Hamburger von der Universität Basel.

\title{
Nichts Neues in der Phytotherapie?
}

Die Hersteller von phytotherapeutischen Präparaten konzentrieren sich auf die Suche nach neuen Indikationen. Als Arzneimittel kommen neue Wirkstoffe nicht auf den Markt, da die regulatorischen Hürden zu hoch sind.

Der Unterschied in der Innovationskraft zwischen den Herstellern schulmedizinischer Präparate und denen von Phytotherapeutika könnte kaum grösser sein. Die Forschungsanstrengungen der grossen Pharmafirmen, die Wirkstoffe für die Schulmedizin herstellen, laufen ständig auf Hochtouren. Eine gut gefüllte Pipeline mit neuen Wirkstoffen gegen immer neue Indikationen ist für die globalen pharmazeutischen Unternehmen wesentlich, um auch in der Zukunft wirtschaftlich überleben $\mathrm{zu}$ können. Zwischen 15 und 20\% ihres Umsatzes werden von der klassischen Pharmaindustrie in Forschung und Entwicklung investiert - ein grosser Teil davon fliesst in die Suche nach neuen patentfähigen Wirkstoffen. Durch die Verfahren der Genomik z.B. sollen sich noch effizientere und mit weniger Nebenwirkungen behaftete Medikamente finden, die den Patienten helfen können.

Anders sieht die Situation bei den Herstellern von phytotherapeutischen Präparaten aus. Hier ist die Suche nach neuen Wirkstoffen kein Geschäftsmodell und Innovationen beschränken sich auf die Suche nach neuen Anwendungsgebieten für Bewährtes. Die Tendenz ist klar, egal welches Produkteportfolio man sich in der Phytotherapeutika-Branche anschaut: Neue Wirkstoffe sind selten zu finden - und selbst nach Veränderungen in der Zusammensetzung von Extrakten, mit denen sich die Wirksamkeit verbessern liesse, sucht man häufig vergebens.

«Die regulatorischen Vorschriften machen die Einführung von neuen
Wirkstoffen oder veränderten $\mathrm{Zu}$ sammensetzungen sehr unattraktiv», sagt Dr. Herbert Schwabl, Verwaltungsratspräsident der Padma AG und Präsident des SVKH (Schweizerischer Verband für Komplementärmedizinische Heilmittel). Neu entdeckte Wirkstoffe oder veränderte Extrakte sorgen dafür, dass die Therapeutika als New Chemical Entities gelten. Die besondere Stellung, die die Pflanzenwirkstoffe als bewährte Volksheilmittel bei Zulassungsbehörden haben, gelten dann nicht mehr. Diese gilt nur, wenn es eine mehr als 30-jährige Erfahrung mit dem pflanzlichen Wirkstoff gibt, davon mehr als 15 Jahre in einem europäischen Land. Statt durch eine vereinfachte $\mathrm{Zu}$ lassung, wie sie in der EU und der Schweiz vorgesehen ist, müssen sie dann ein aufwendiges Registrierungsverfahren durchlaufen. «Das ist einer der wichtigsten Gründe, warum kaum neue pflanzliche Wirkstoffe untersucht werden", sagt Schwabl. Das unternehmerische Risiko ist viel zu gross. Der Nachteil: Innovationen sind nicht möglich.

Die Entwicklung neuer Wirkstoffe mit der strengen Registrierung ist ohne die gut gefüllten Schatullen eines grossen Konzerns kaum zu schaffen. Selbst für sie sind die finanziellen Lasten immens. Novartis-Chef Joe Jimenez beziffert die Kosten für ein neues Medikament auf mittlerweile eine Milliarde Franken und macht die immer weiter steigenden regulatorischen Ansprüche dafür verantwortlich. Auch wenn der absolute Aufwand bei der Entwicklung von phytotherapeutischen Präparaten 


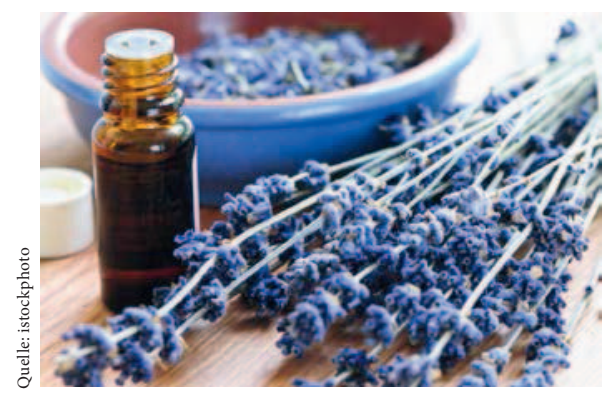

geringer ist, gehen die Kosten für ein komplettes Zulassungsverfahren dennoch schnell in die Millionen. Das Risiko des Scheiterns ist aber immer noch sehr hoch, weil sich selbst viel versprechende Mittel in den letzten klinischen Studien noch als wenig wirkungsvoll oder unsicher erweisen. Kaum ein mittelständisches Unternehmen kann sich ein solches Wagnis leisten - dass kaum Wirkstoffinnovationen auf den Markt kommen, verwundert deshalb nicht.

Grosse Hoffnungen setzt der SVKH deshalb auf die Revision des Heilmittelgesetzes. Mit ihr könnte die aufwendige Registrierung der plötzlich als New Chemical Entities eingestuften Pflanzenwirkstoffe hinfällig werden. Für die Unternehmen wäre das eine grosse Erleichterung, und das entscheidende Innovationshindernis würde entfallen. Der SVKH hat den Bundesrat gebeten, die vereinfachte Zulassung zu konkretisieren, da der Ermessensspielraum der Heilmittel-Zulassungsbehörde Swissmedic zu gross sei: Selbst die vereinfachte Zulassung sei komplex und teuer geworden. «Die Hürden sind so hoch, dass jährlich viele Produkte vom Markt verschwinden, dagegen kaum neue Produkte zugelassen werden», sagt Schwabl. Nach seinen Vorstellungen soll bei Heilmitteln, die seit vielen Jahren im Westen auf dem Markt sind, auf die pharmakologische, toxikologische und klinische Prüfung verzichtet werden. Die langjährige Anwendungspraxis hat diese dann ja schon erbracht.

«Wir konzentrieren uns darauf, für bewährte Wirkstoffe und Extrakte neue Indikationen zu finden», sagt
Dr. Günter Meng, der bei Schwabe für die Forschung und Entwicklung zuständig ist. Das Unternehmen hat in den letzten Jahren intensiv geforscht und den Einsatz von Lavendel bei Angststörungen getestet. Schon seit Jahren hat es Produkte auf der Basis von Lavendel im Programm. Für den Einsatz bei Angststörungen hat Lasea ${ }^{\circledR}$ im vergangenen Jahr die $\mathrm{Zu}$ lassung in Deutschland bekommen. In der Schweiz ist das Lavendelölpräparat für diesen Einsatz noch nicht zugelassen. Die Strategie, statt neuer Wirkstoffe neue Indikationen für Bewährtes zu finden, ist vom finanziellen Aufwand auch nicht zu unterschätzen. Für die Registrierung müssen trotzdem umfangreiche Studien durchgeführt werden, die ihren Preis haben: Etwa $10-15 \%$ des Umsatzes werden auch bei den Herstellern für phytotherapeutische Präparate für die Forschung und Entwicklung aufgewendet. Der Anteil lässt sich durchaus mit dem der klassischen Pharmaunternehmen vergleichen. Bei Schwabe konzentriert man sich nun auf die Vermarktung von Lasea und hält sich bedeckt, wenn es um andere bewährte Wirkstoffe geht, für die man jetzt neue Indikationen sucht. "Wir kümmern uns jetzt erst einmal darum, dass Lasea bei der neuen Indikation ein Erfolg wird», sagt Meng.

Auf der Suche nach erweiterten Einsatzmöglichkeiten bedienen sich auch die Phytofirmen moderner biologischer Analyseverfahren. Auf molekularer Ebene werden z.B. Rezeptoren gesucht, mit denen pflanzliche Wirkstoffe interagieren können. Die Ethnomedizin spielt dabei kaum eine Rolle, denn der Transfer von solchem Wissen scheitert häufig an der Kommunikation: Oft ist unklar, von welchen Krankheiten z.B. Medizinmänner sprechen, weil sich die Definition von Krankheiten von Kultur zu Kultur unterscheidet. Oft ist nicht klar, welche Pflanzen gemeint sind und wie die Substanzen zubereitet werden. Weil bei der Verwendung von tradiertem Wissen die Völker der Ursprungsregion nach dem RioProtokoll zur Biodiversität finanziell entschädigt werden müssen, kommt es zu rechtlichen Stolpersteinen: Unterschiedliche Auffassungen, was ein Vertrag ist oder welche Konsequenzen damit verbunden sind, haben schon einige europäische Phytohersteller den Rückzug vom Feld der Ethnomedizin antreten lassen.

Auch an den Universitäten ist die Forschung nach neuen Pflanzenwirkstoffen auf dem Rückzug. Der Münsteraner Professor für Pharmazeutische Biologie und Phytochemie Andreas Hensel malte auf dem «Phytotherapeutika»-Kongress 2012 in Wien ein düsteres Bild von der Zukunft der Wirkstoffforschung an Pflanzen. An nur 7 von über 20 Universitäten mit Pharmazieinstituten in Deutschland wird Arzneimittelforschung an Pflanzen betrieben. Einige Lehrstühle werden nicht mehr neu besetzt, wenn die heutigen Inhaber emeritiert werden, und einige Institute sind von der Schliessung bedroht. In der Schweiz wird an den Universitäten Basel und Zürich sowie in Wädenswil an der ZHAW (Zürcher Hochschule für Angewandte Wissenschaften) über Phytotherapie geforscht.

Neben Schwabe suchen auch andere Hersteller von Phytotherapeutika nach neuen Einsatzmöglichkeiten für ihre bewährten Präparate. Die Padma AG geht dabei den molekulargenetischen Weg. «Wir erforschen, wo Padma 28 bei chronischen Entzündungen auf der Ebene der Gene wirkt», sagt Herbert Schwabl. «Es scheint, dass die tibetische Rezeptur bei vielen Alterskrankheiten eine Rolle spielt, indem sie ihnen den Nährboden entzieht.» Ende September hat das Unternehmen in Zusammenarbeit mit dem Institut für Naturheilkunde der Universität Zürich einen Kongress veranstaltet, in dessen Rahmen die ersten Ergebnisse dieser Untersuchungen vorgestellt wurden. «Für Firmen ist es wichtig, dass sie ihre bestehenden Präparate im Markt halten können», 
sagt Schwabl. Die immer schärferen Sicherheitsanforderungen sorgen dafür, dass viele Untersuchungen durchgeführt werden müssen, damit die Registrierung nicht entzogen wird. Die seit dem letzten Jahr geltenden Richtlinien der Europäischen Union für die Zulassung von pflanzlichen Präparaten haben das Ziel, die Konsumentinnen und Konsumenten zu schützen. Sie sorgen aber auch dafür, dass bisher etablierte Präparate vom Markt genommen werden müssen, wenn sie die neuen Anforderungen nicht erfüllen. So führte etwa die Überprüfung der Indikationen für Kinder dazu, dass Fenchel als Arzneimittel für Kinder als nicht geeignet eingestuft wird, während er in Lebensmitteln und als Tee sehr wohl zu kaufen ist. Für den Schweizer Markt haben diese Bestimmungen keine direkten Auswirkungen, bestimmen aber die Chancen auf eine Zulassung künftig indirekt mit. Für die eigenständige Schweizer Tradition der Naturheilkunde sind deswegen angepasste gesetzliche Bestimmungen von grösster Wichtigkeit.

\section{Neue Indikationen in der Neurologie}

Wenn schon keine neuen Wirkstoffe ihren Weg in die Regale finden, werden von den Herstellern Indikationen erschlossen, für die es bisher keine zugelassenen Behandlungen mit Phytotherapeutika gab. Die Erweiterung der Indikationen der bewährten Wirkstoffe geht vor allem in die Richtung der neuronalen Erkrankungen und der Behandlung von Befindlichkeitsstörungen. Mit der Konzentration auf das Nervensystem tragen die Hersteller phytotherapeutischer Präparate der Entwicklung der Demographie in Europa Rechnung: Durch die im Schnitt immer älter werdende Bevölkerung wächst der Bedarf an Alzheimer- oder Demenzpräparaten.
Jedoch finden auch Wirkstoffe und Extrakte, die die Konzentration verbessern oder das Gedächtnis fördern, grosses Interesse. Weil sich immer mehr Menschen beruflich und privat unter Druck fühlen, nehmen auch die psychischen Beschwerden zu: Die Gemütsstimmung leidet darunter, Menschen empfinden Angst. Kein Wunder, dass Präparate zur Behandlung von Befindlichkeitsstörungen auf grosse Resonanz stossen. «Bei Lasea haben wir sehr gute Erfahrungen gemacht», sagt Günter Meng, Forschungs- und Entwicklungschef bei Schwabe.

Die Konzentration der Hersteller phytotherapeutischer Präparate auf Befindlichkeitsstörungen und andere psychische Indikationen ist nicht nur wirtschaftlich sinnvoll. Es sind Anwendungen, bei denen sich die Behandlung mit der Phytotherapie aufgrund der Ursachen der Beschwerden besonders anbietet. Weil in den Extrakten oft eine grosse Zahl an wirksamen Substanzen vertreten ist, die entweder allein oder in Kombination wirken, scheinen die Präparate

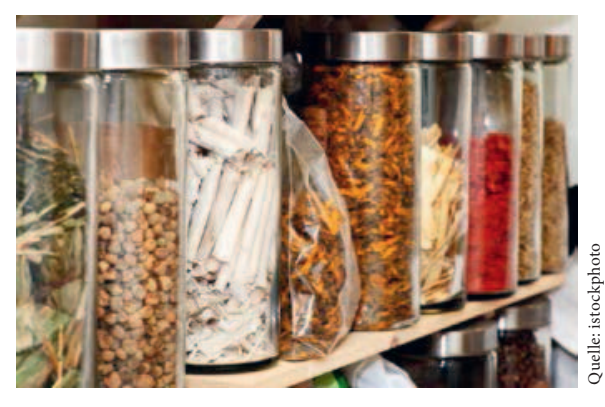

für die Behandlung von psychischen Beschwerden besonders gut geeignet $\mathrm{zu}$ sein. Diese können eine Reihe von Ursachen haben, die von Patient zu Patient unterschiedlich sein können - ein natürlicher Cocktail, in dem sich viele wirksame Substanzen befinden, verspricht hier die grösste Wirkung. Der Einsatz von Pflanzen, um die Stimmung und Wahrnehmung der Wirklichkeit zu beeinflussen, hat eine lange Tradition. Bereits im 3. Jahrhundert vor Christus beschrieb Theophrastos von Eresos die psychoaktive Wirkung von Pflanzen (siehe Kasten). So greifen die Innovationen in der Phytotherapie das alte Erbe der Antike wieder auf. (rfi)

\section{Der Innovationsschub in der Renaissance}

Der Stillstand bei der Entdeckung und Anwendung neuer wirksamer Substanzen wirkt erschreckend, wenn man ihn mit der wissenschaftlichen Neugier vergleicht, durch die sich die Pflanzenheilkunde einmal ausgezeichnet hat. Immer war man auf der Suche nach neuen Pflanzen, mit denen sich Erkrankungen behandeln liessen. Hippokrates nennt im 4. Jahrhundert v. Chr. etwa 230 Pflanzen, die sich für Heilzwecke verwenden lassen. Theophrastos von Eresos kennt 100 Jahre später bereits 500 solcher Pflanzen. Bei den Römern führen Gelehrte wie Plinius
600 Heilpflanzen. Im Mittelalter findet z.B. aus den Schriften des persischen Arztes Avicenna zusätzliches Heilwissen seinen Weg nach Europa. In seinem Kräuterbuch von 1625 nennt Jacobus Theodorus über 3000 Heilpflanzen. Vor mehr als 500 Jahren ist die Pflanzenmedizin Neuem gegenüber sehr aufgeschlossen gewesen. Einer der Väter der modernen Botanik und Pflanzenheilkunde, Leonardus Fuchs, warnte davor, sich auf das altbewährte Wissen zu beschränken. Viel besser sei es, die Natur zu studieren, um Neues $\mathrm{zu}$ finden. 